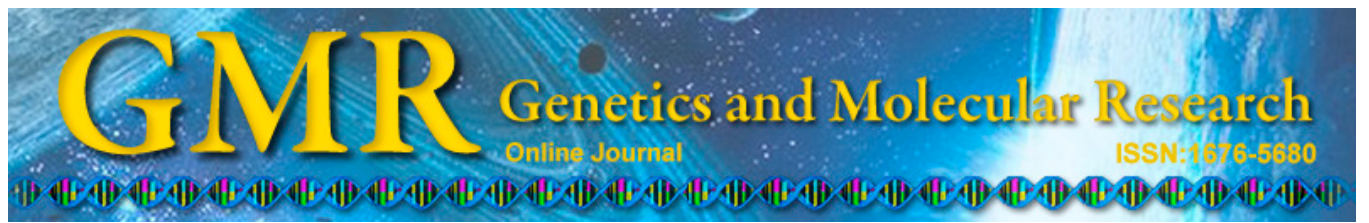

\title{
Development and cross-species transferability of unigene-derived microsatellite markers in an edible oil woody plant, Camellia oleifera (Theaceae)
}

\author{
B.G. Jia ${ }^{1,2}$, Q. Lin $^{1,2}$, Y.Z. Feng ${ }^{3}$, X.Y. Hu ${ }^{4}$, X.F. Tan ${ }^{1,2}$, F.G. Shao ${ }^{1,2}$ and \\ L. Zhang ${ }^{1,2}$
}

${ }^{1}$ Key Laboratory of Cultivation and Protection for Non-Wood Forest Trees, Ministry of Education, Central South University of Forestry and Technology, Changsha, Hunan, China

${ }^{2}$ Cooperative Innovation Center of Cultivation and Utilization for Non-Wood Forest Trees of Hunan Province, Central South University of Forestry and Technology, Changsha, Hunan, China

${ }^{3}$ China Paulownia Research Center, Zhengzhou, Henan, China

${ }^{4}$ School of Forestry and Landscape Architecture, Anhui Agricultural University, Hefei, Anhui, China

Corresponding author: L. Zhang

E-mail: triwoodtim918@126.com

Genet. Mol. Res. 14 (2): 6906-6916 (2015)

Received December 18, 2014

Accepted April 8, 2015

Published June 18, 2015

DOI http://dx.doi.org/10.4238/2015.June.18.33

\begin{abstract}
Camellia oleifera is an important edible oil woody plant in China. Lack of useful molecular markers hinders current genetic research on this tree species. Transcriptome sequencing of developing $C$. oleifera seeds generated 69,798 unigenes. A total of 6949 putative microsatellites were discovered among 6042 SSRcontaining unigenes. Then, 150 simple sequence repeats (SSRs) were evaluated in 20 varieties of $C$. oleifera. Of these, 52 SSRs revealed
\end{abstract}


polymorphism, with the number of alleles per locus ranging from 2 to 15 and expected heterozygosity values from 0.269 to 0.888 . The polymorphic information content varied from 0.32 to 0.897 . Crossspecies transferability rates in Camellia chekangoleosa and Camellia japonica were 90.4 and $78.8 \%$, respectively. The 52 polymorphic unigene-derived SSR markers serve to enrich existing microsatellite marker resources for $C$. oleifera and offer potential for applications in genetic diversity evaluation, molecular fingerprinting, and genetic mapping in C. oleifera, C. chekangoleosa, and C. japonica.

Key words: Camellia oleifera; $C$. chekangoleosa; $C$. japonica; Unigene; Microsatellite; Cross-species transferability

\section{INTRODUCTION}

The woody plant Camellia oleifera, a member of the Theaceae family, is economically important for the production of tea oil in China. Tea oil, extracted from the seeds, is considered a high-quality edible oil and is also called "eastern olive oil" because its composition is highly similar to that of olive oil (Ma et al., 2011). Oil-tea trees include more than ten species, of which C. oleifera is the dominant species in tea oil production, being the most widely distributed and planted in China and producing the highest yields. A number of authorized elite varieties have so far been released for tea oil production. However, more new varieties with novel traits, such as high quality, multipurpose use, and biotic and abiotic stress resistance, are required for commercial plantations. Understanding the genetic diversity and relatedness among germplasm resources is useful for breeding and clonal improvements. Microsatellite markers, also called simple sequence repeat (SSR) markers, are powerful tools for genetic diversity evaluation, molecular fingerprinting, and genetic mapping. In the past few years, transcriptome sequencing technology has developed to offer a fast, cost-effective, and reliable approach to the generation of large expression-data sets, in both model and non-model plants with large, complex genomes (Marioni et al., 2008; Mortazavi et al., 2008; Nagalakshmi et al., 2008). In addition, this technology provides an opportunity to identify and develop unigene-derived microsatellite markers (Gupta and Gopalakrishna, 2010; Dutta et al., 2011; Zhang et al., 2012). These new markers are considered superior to genomic SSR markers because they potentially code for functional proteins and can increase the efficiency of marker-assisted selection.

Recently, microsatellites have been analyzed and developed in Camellia chekangoleosa, an allied species of C. oleifera also used for tea oil production (Wen et al., 2012; Shi et al., 2013). C. chekangoleosa is a diploid species, whereas different ploidy levels occur in C. oleifera (Huang, 2013). C. oleifera-derived specific microsatellite markers have not yet been reported, hindering genetic research efforts. In our previous study, we performed Illumina platform-based transcriptome sequencing of developing $C$. oleifera seeds in order to understand seed fatty acid metabolism (Shao, 2011). The objective of this study was to characterize microsatellites from the transcriptome sequences and develop polymorphic microsatellite markers in C. oleifera. These novel unigene-derived microsatellite markers will provide a useful tool for genetic research and comparative genome analysis in C. oleifera and allied species. 


\section{MATERIAL AND METHODS}

\section{Plant materials}

Plant materials used for validation of SSRs comprised 20 varieties of C. oleifera, including the 'Huashuo' cultivar, which we utilized for complementary DNA (cDNA) library construction and transcriptome sequencing. In addition, 18 elite germplasm clones of C. chekangoleosa and 15 varieties of Camellia japonica were used to investigate crossspecies transferability. All plants were conserved at the Germplasm Repository of the Jiangxi Academy of Forestry, Nanchang, China. Young leaves were collected in spring and stored at $-80^{\circ} \mathrm{C}$ until use.

\section{Source of transcriptome sequences}

Our laboratory, in collaboration with Beijing Genomics Institute in Shenzhen, China, constructed a cDNA library using developing seeds of the 'Huashuo' cultivar at the lipid synthesis initiation phase and the peak lipid synthesis phase, i.e., 180 and 300 days after flowering, respectively. Detailed procedures for cDNA library construction, sequencing, and de novo transcriptome assembly were described previously (Shao, 2011).

\section{Detection of microsatellites and designing of SSR primers}

Microsatellites were detected using the Microsatellite (MISA) tool, with parameters set for detection of perfect di-, tri-, tetra-, penta-, and hexanucleotide motifs with at least six, five, five, four, and four repeats, respectively. Primer pairs were designed using Primer Premier 5.0. The major parameters for primer design were set as follows: SSR motifs $\geq 20 \mathrm{bp}$, primer length from 18 to 25 nucleotides, and PCR product size from 100 to $300 \mathrm{bp}$ (Table 1).

\section{Validation of microsatellite markers}

Genomic DNA was isolated from young leaves of 20 C. oleifera varieties, 18 C. chekangoleosa clones, and 15 C. japonica varieties using a DNA isolation kit (Tiangen Biotech, China). Of the 20 C. oleifera varieties, three were first used to test the primers. Each PCR was carried out in a total volume of $10 \mu \mathrm{L}$ containing $1 \mathrm{X}$ buffer, $2 \mathrm{mM} \mathrm{MgCl}_{2}, 0.2 \mathrm{mM}$ of each dNTPs, $0.2 \mu \mathrm{M}$ of each primer, 0.5 U Taq DNA polymerase (Tiangen Biotech), and 10 ng DNA template. PCR was performed on a thermal cycler (ABI9700, Applied Biosystems, USA) under the following conditions: $94^{\circ} \mathrm{C}$ for $5 \mathrm{~min} ; 30$ cycles of $30 \mathrm{~s}$ at $94^{\circ} \mathrm{C}, 45 \mathrm{~s}$ at $56^{\circ} \mathrm{C}$, and $45 \mathrm{~s}$ at $72^{\circ} \mathrm{C} ; 10$ cycles of $30 \mathrm{~s}$ at $94^{\circ} \mathrm{C}, 45 \mathrm{~s}$ at $53^{\circ} \mathrm{C}$, and $45 \mathrm{~s}$ at $72^{\circ} \mathrm{C}$; and a final extension of $5 \mathrm{~min}$ at $72^{\circ} \mathrm{C}$. The amplified products were analyzed on $2 \%$ agarose gels. Any loci generating products of the expected size were then assessed for polymorphisms in the 20 C. oleifera varieties, using the M13 (-21) (5'-TGTAAAACGACGGCCAGT-3') sequence-tag method (Schuelke, 2000). Fluorescently labeled PCR products were analyzed concurrently with the GeneScan-500 LIZ Size Standard on an ABI 3730XL sequencer, and sizes were determined with GeneMapper v4.0. 
Camellia oleifera SSR Markers

6909

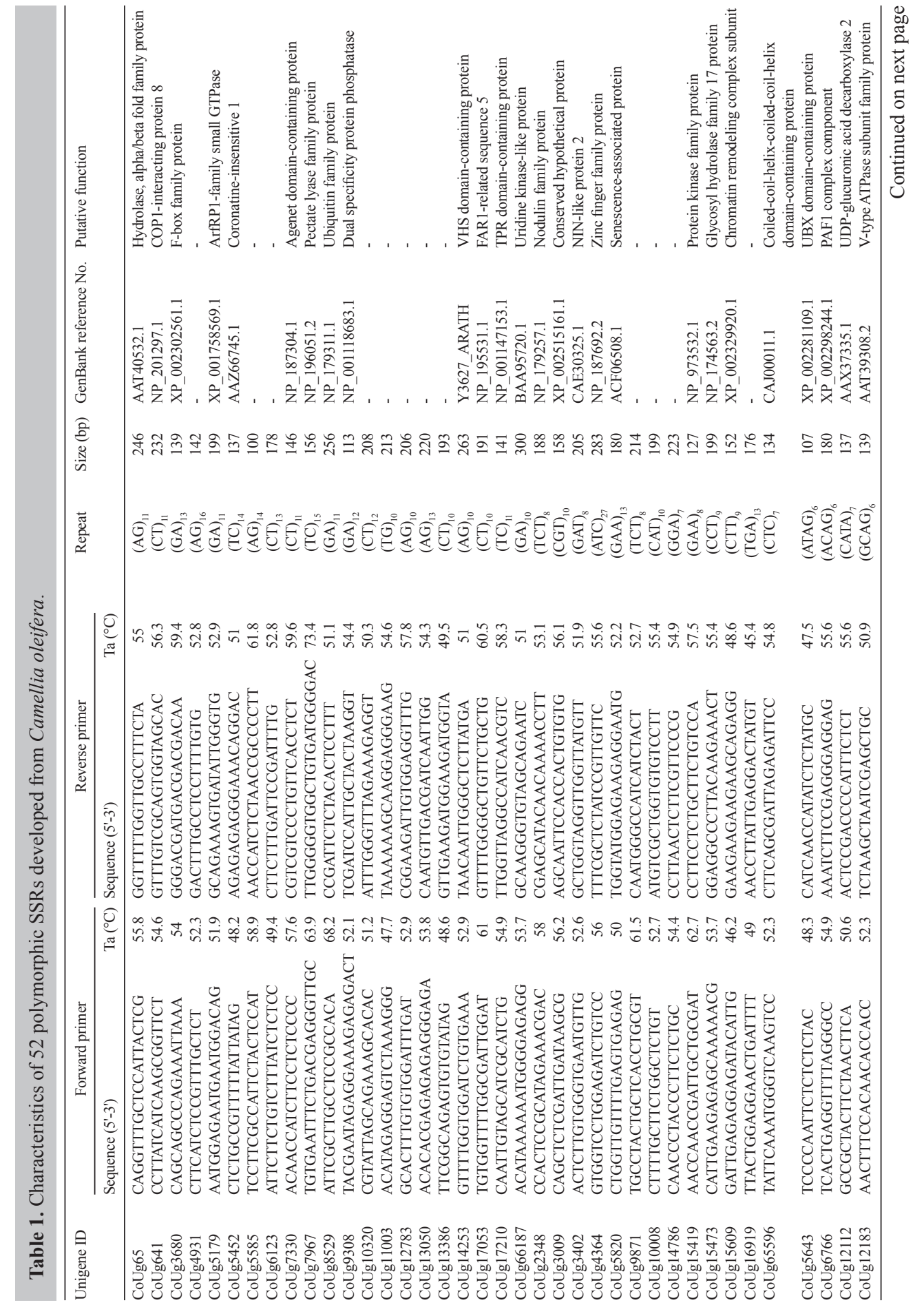


B.G. Jia et al.

6910

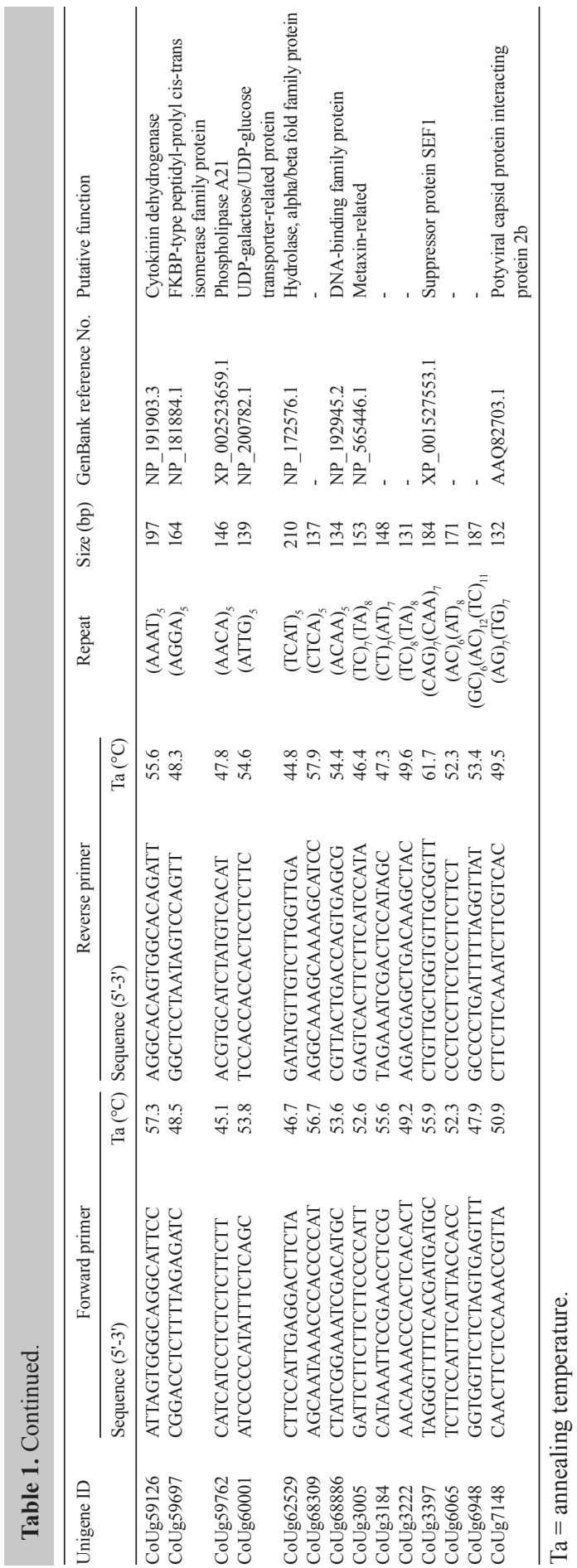




\section{Statistical analysis}

Due to the putatively polyploid character of C. oleifera, statistical analysis entailed treating the SSRs as dominant markers. The data were scored in a binary format (presence as ' 1 ' and absence as ' 0 '). The binary data were used to calculate genetic parameters using PopGene v1.32. The polymorphism information content (PIC) value for each SSR marker was calculated as described previously (Huang, 2013; Singh et al., 2013). For C. chekangoleosa (diploid) and C. japonica (diploid), statistical analysis treated the SSRs as codominant markers.

\section{RESULTS}

\section{Characterization of microsatellites in the $C$. oleifera unigenes}

Transcriptome sequencing of developing seeds of $C$. oleifera produced 69,798 unigenes, which were used to identify microsatellites with the MISA tool. A total of 6949 putative SSR motifs from 6042 SSR-containing unigenes were identified; that is, $9.9 \%$ of the unigenes contained at least one of the considered microsatellites. Dinucleotide repeats were the most common types of SSR, accounting for $64.8 \%$, followed by trinucleotide (26.1\%), hexanucleotide $(5 \%)$, pentanucleotide $(2.6 \%)$, and tetranucleotide $(1.5 \%)$ repeats (Figure 1$)$.

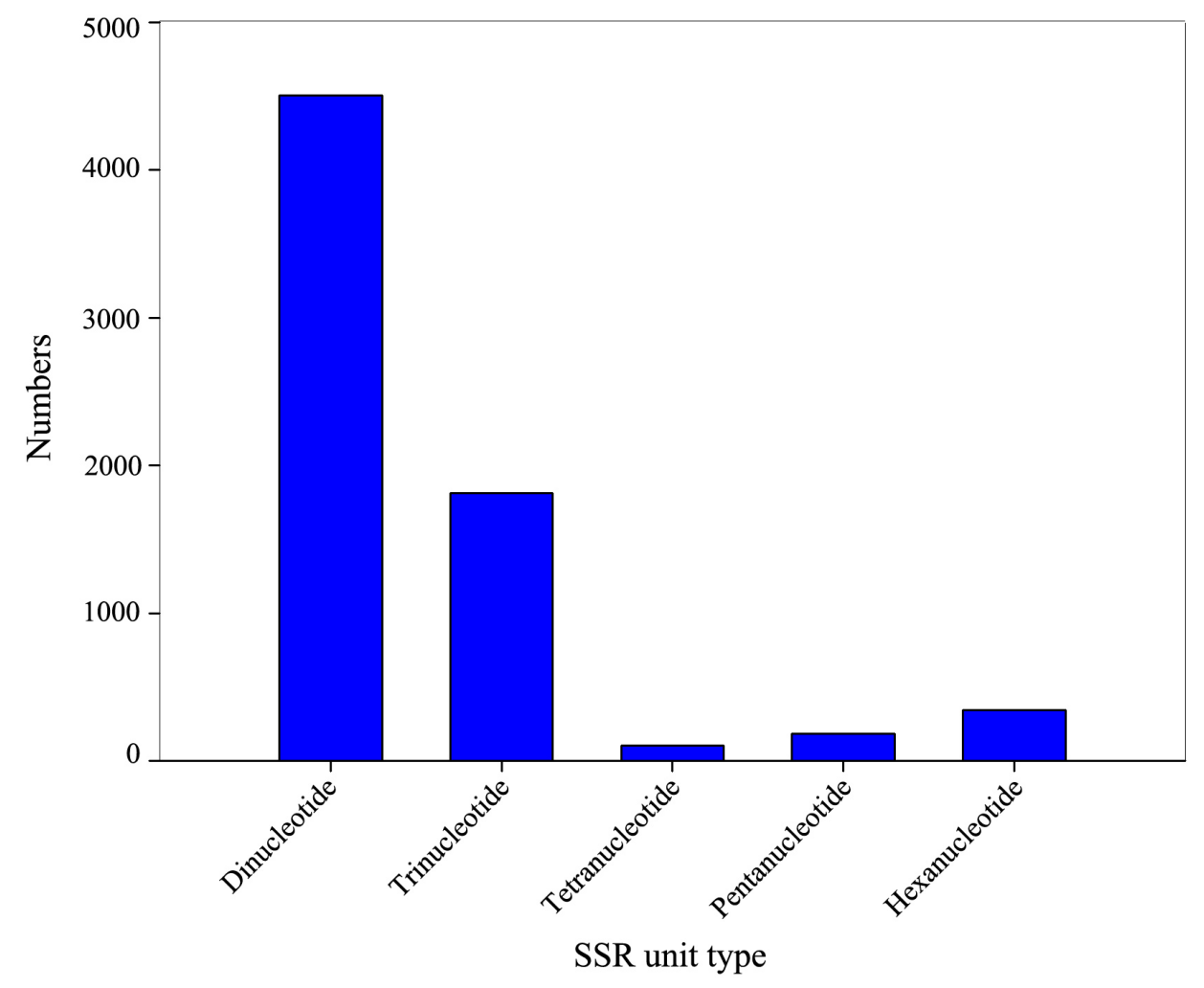

Figure 1. Distribution of different SSR unit types in Camellia oleifera. 
The most abundant SSR motif was AG/CT, followed by AAG/CTT, AT/AT, AC/GT, ACC/GGT, ATC/ATG, AGG/CCT, AAC/GTT, and AGC/CTG (Figure 2). Among the dinucleotide repeats, the motif AG/CT was the most common (84.5\%), followed by AT/TA (8.5\%) and $\mathrm{AC} / \mathrm{GT}(6.9 \%)$. Among the trinucleotide repeats, the motif AAG/CTT was the most common, accounting for $30.2 \%$, followed by ACC/GGT (16.3\%) and ATC/ATG (15.5\%). Other motifs were identified in insignificant numbers.

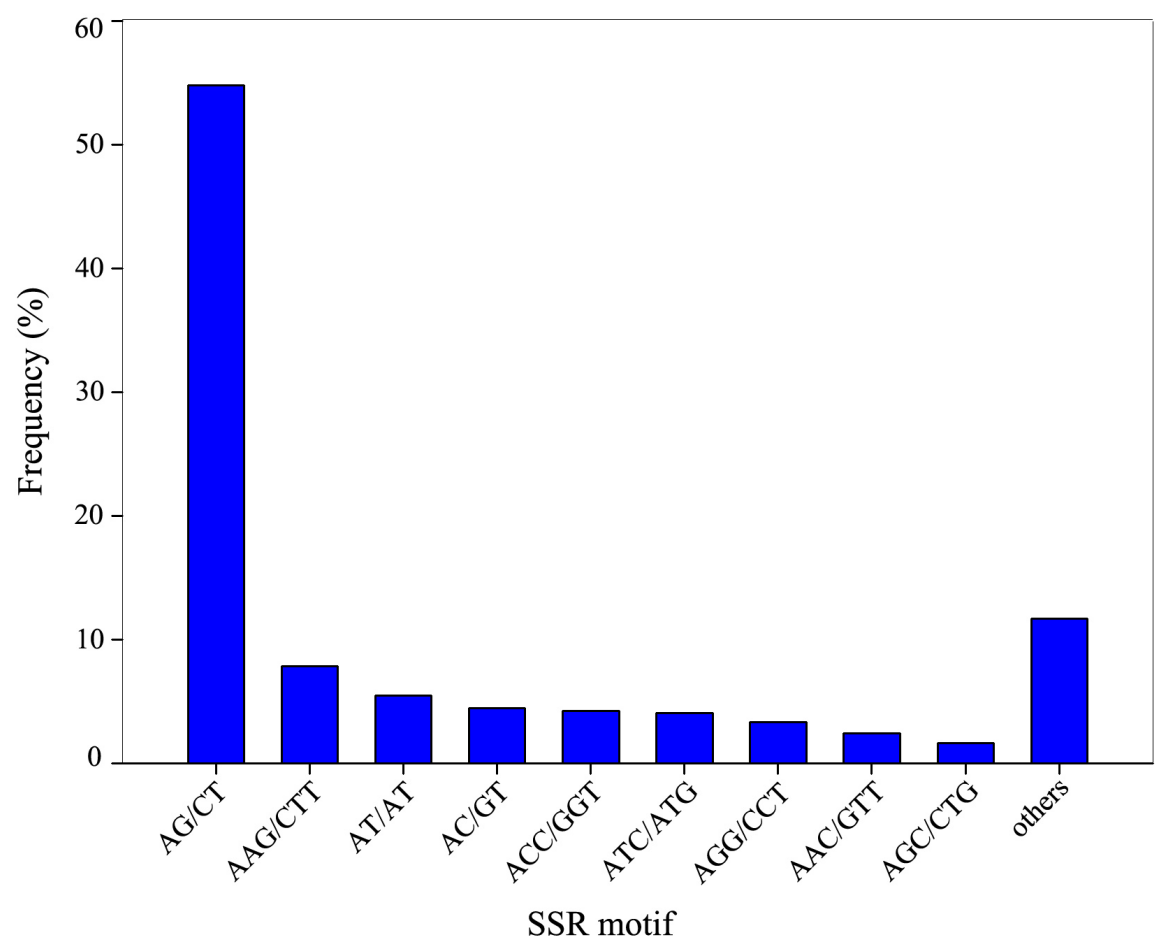

Figure 2. Frequency of different SSR motifs in Camellia oleifera.

\section{Development of $C$. oleifera polymorphic SSRs}

One hundred and fifty primer pairs, corresponding to 150 unigene loci, were designed for the PCR test. Initial amplification was conducted on three $C$. oleifera varieties with unlabeled primers. The amplified products were analyzed using agarose gel electrophoresis. Thirty-two loci generated nonspecific products and 16 loci yielded no products. The remaining 102 loci yielded products of expected size and were then assessed for polymorphism in $20 \mathrm{C}$. oleifera varieties with the M13 (-21) (5'-TGTAAAACGACGGCCAGT-3') sequence-tag method.

As shown by capillary electrophoresis, PCR products appearing as one band on an agarose gel were clearly separated into four distinct fragments by capillary electrophoresis (Figure 3A). Based on capillary electrophoresis data, 52 loci among the C. oleifera varieties displayed polymorphism tested (Table 1). The number of alleles $\left(N_{\mathrm{A}}\right)$ per locus ranged from 2 to 15 , with an average of 7.096 . The expected heterozygosity $\left(H_{\mathrm{E}}\right)$ value ranged from 0.32 to 0.897 , with an average of 0.707 . The PIC ranged from 0.498 to 0.887 , with an average 
of 0.742 . Of the 52 loci, 49 were high polymorphic (PIC $>0.5)$ and only three (CoUg3898, CoUg12183, and CoUg14786) were moderately polymorphic $(0.25<$ PIC $<0.5)$ (Table 2$)$.

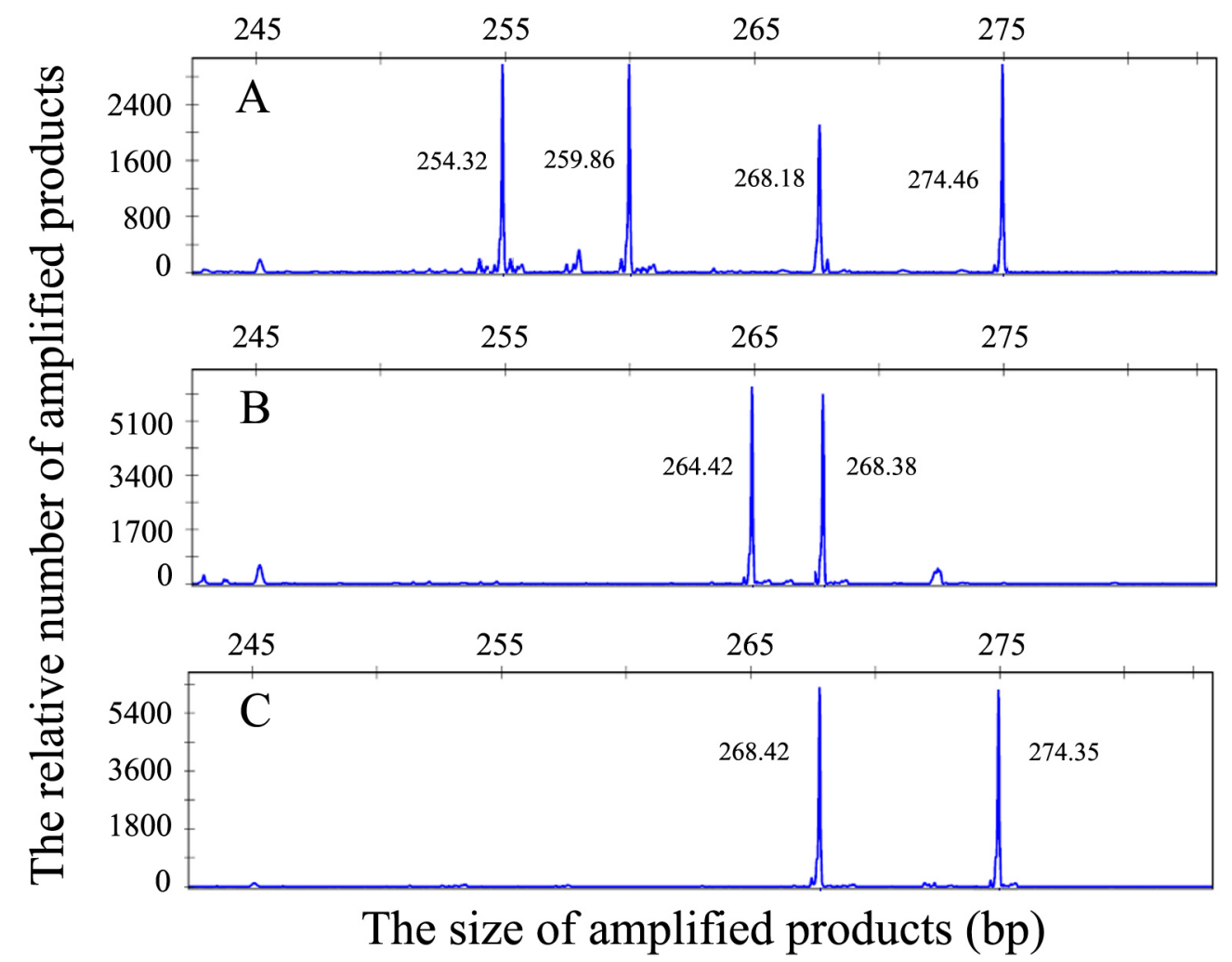

Figure 3. Analysis of fluorescently labeled PCR products by capillary electrophoresis.

\section{Cross-species transferability of the $C$. oleifera SSRs}

Cross-amplification tests were performed in 18 elite germplasm clones of C. chekangoleosa and 15 varieties of $C$. japonica, both of which are diploid species in Camellia. The SSR markers from C. oleifera produced at most two alleles in each sample of the two species (Figure 3B and C). For C. chekangoleosa, 47 SSR markers exhibited polymorphisms and the crossspecies transferability rate was $90.4 \%$. The $N_{\mathrm{A}}$ ranged from 2 to 10 , with an average of 5.319 per locus. The $H_{\mathrm{E}}$ ranged from 0.056 to 0.873 , with an average of 0.672 . The PIC value ranged from 0.1 to 0.862 , with an average of 0.67 . Of the 47 markers, 42 were highly polymorphic, one was moderately polymorphic, and four had low polymorphism $(\mathrm{PIC}<0.25)$ (Table 2$)$.

For $C$. japonica, 41 markers exhibited polymorphisms and the cross-species transferability rate was $78.8 \%$. The $N_{\mathrm{A}}$ ranged from 2 to 7 , with an average of 4.61 per locus. The $H_{\mathrm{E}}$ ranged from 0.067 to 0.86 , with an average of 0.652 . The PIC value ranged from 0.117 to 0.831 , with an average of 0.649 . Of the 41 markers, 36 were highly polymorphic, one was moderately polymorphic, and four had low polymorphism (Table 2). These results indicated that the 52 SSR markers developed from C. oleifera harbored rich polymorphisms in the other Camellia plants. 
B.G. Jia et al.

\begin{tabular}{|c|c|c|c|c|c|c|c|c|c|c|c|c|}
\hline \multirow[t]{2}{*}{ Unigene ID } & \multicolumn{4}{|c|}{ C. oleifera $(\mathrm{N}=20)$} & \multicolumn{4}{|c|}{ C. chekangoleosa $(\mathrm{N}=18)$} & \multicolumn{4}{|c|}{ C. japonica $(\mathrm{N}=15)$} \\
\hline & $N_{\mathrm{A}}$ & $H_{\mathrm{o}}$ & $H_{\mathrm{E}}$ & PIC & $N_{\mathrm{A}}$ & $H_{\mathrm{o}}$ & $H_{\mathrm{E}}$ & PIC & $N_{\mathrm{A}}$ & $H_{\mathrm{o}}$ & $H_{\mathrm{E}}$ & PIC \\
\hline CoUg65 & 8 & 1.000 & 0.834 & 0.852 & 7 & 0.824 & 0.857 & 0.835 & 7 & 0.857 & 0.860 & 0.831 \\
\hline CoUg641 & 9 & 0.900 & 0.850 & 0.865 & 4 & 0.222 & 0.671 & 0.661 & 4 & 0.200 & 0.660 & 0.648 \\
\hline CoUg3680 & 6 & 0.611 & 0.657 & 0.702 & 6 & 0.500 & 0.765 & 0.765 & 6 & 0.467 & 0.766 & 0.760 \\
\hline CoUg4931 & 10 & 0.684 & 0.870 & 0.882 & 7 & 0.706 & 0.752 & 0.756 & 6 & 0.643 & 0.725 & 0.707 \\
\hline CoUg5179 & 14 & 0.950 & 0.881 & 0.890 & 10 & 0.833 & 0.851 & 0.826 & - & - & - & - \\
\hline CoUg5452 & 10 & 0.737 & 0.853 & 0.867 & 7 & 0.389 & 0.821 & 0.829 & 6 & 0.333 & 0.816 & 0.800 \\
\hline CoUg5585 & 10 & 1.000 & 0.839 & 0.856 & 8 & 0.722 & 0.803 & 0.803 & - & - & - & - \\
\hline CoUg6123 & 15 & 0.800 & 0.888 & 0.897 & - & - & - & - & - & - & - & - \\
\hline CoUg7330 & 8 & 0.700 & 0.796 & 0.819 & 4 & 0.111 & 0.686 & 0.695 & 4 & 0.133 & 0.745 & 0.734 \\
\hline CoUg7967 & 6 & 0.650 & 0.768 & 0.798 & 4 & 0.278 & 0.618 & 0.631 & 3 & 0.267 & 0.559 & 0.598 \\
\hline CoUg8529 & 8 & 0.900 & 0.843 & 0.859 & 8 & 0.941 & 0.863 & 0.837 & - & - & - & - \\
\hline CoUg9308 & 10 & 0.842 & 0.837 & 0.853 & 6 & 0.412 & 0.733 & 0.747 & 6 & 0.500 & 0.706 & 0.730 \\
\hline CoUg10320 & 8 & 0.611 & 0.827 & 0.846 & 5 & 0.333 & 0.684 & 0.719 & 5 & 0.400 & 0.743 & 0.748 \\
\hline CoUg11003 & 5 & 0.278 & 0.657 & 0.712 & 5 & 0.177 & 0.786 & 0.775 & 5 & 0.214 & 0.802 & 0.782 \\
\hline CoUg12783 & 12 & 0.900 & 0.852 & 0.866 & 7 & 0.444 & 0.829 & 0.825 & 7 & 0.467 & 0.825 & 0.826 \\
\hline CoUg13050 & 3 & 0.067 & 0.559 & 0.539 & - & - & - & - & - & - & - & - \\
\hline CoUg13386 & 5 & 0.842 & 0.669 & 0.714 & 4 & 0.333 & 0.722 & 0.691 & 4 & 0.267 & 0.747 & 0.709 \\
\hline CoUg14253 & 8 & 0.450 & 0.766 & 0.793 & 5 & 0.059 & 0.711 & 0.710 & 5 & 0.071 & 0.638 & 0.649 \\
\hline CoUg17053 & 7 & 0.500 & 0.761 & 0.791 & 5 & 0.177 & 0.640 & 0.665 & 4 & 0.143 & 0.564 & 0.602 \\
\hline CoUg17210 & 5 & 0.667 & 0.668 & 0.714 & 7 & 0.222 & 0.833 & 0.826 & 7 & 0.200 & 0.832 & 0.821 \\
\hline CoUg66187 & 3 & 0.150 & 0.492 & 0.552 & 6 & 0.529 & 0.763 & 0.754 & 5 & 0.357 & 0.738 & 0.737 \\
\hline CoUg2348 & 8 & 0.750 & 0.745 & 0.776 & 6 & 0.000 & 0.775 & 0.753 & 4 & 0.000 & 0.726 & 0.702 \\
\hline CoUg3009 & 5 & 0.900 & 0.705 & 0.746 & 7 & 0.833 & 0.754 & 0.749 & 7 & 0.800 & 0.759 & 0.752 \\
\hline CoUg3402 & 7 & 0.850 & 0.623 & 0.678 & 2 & 0.059 & 0.166 & 0.198 & - & - & - & - \\
\hline CoUg4364 & 11 & 1.000 & 0.859 & 0.872 & 4 & 0.059 & 0.668 & 0.570 & 4 & 0.071 & 0.664 & 0.658 \\
\hline CoUg5820 & 9 & 1.000 & 0.813 & 0.833 & - & - & - & - & - & - & - & - \\
\hline CoUg9871 & 7 & 0.900 & 0.732 & 0.767 & 8 & 0.889 & 0.813 & 0.792 & - & - & - & - \\
\hline CoUg10008 & 9 & 0.900 & 0.780 & 0.801 & 5 & 0.111 & 0.752 & 0.740 & 5 & 0.067 & 0.738 & 0.719 \\
\hline CoUg14786 & 2 & 0.053 & 0.269 & 0.320 & 3 & 0.000 & 0.585 & 0.567 & 3 & 0.000 & 0.540 & 0.520 \\
\hline CoUg15419 & 3 & 0.706 & 0.418 & 0.523 & 2 & 0.167 & 0.157 & 0.245 & 2 & 0.133 & 0.129 & 0.208 \\
\hline CoUg15473 & 4 & 0.895 & 0.522 & 0.603 & 3 & 0.118 & 0.116 & 0.194 & 2 & 0.143 & 0.138 & 0.219 \\
\hline CoUg15609 & 4 & 0.800 & 0.585 & 0.651 & 2 & 0.056 & 0.056 & 0.100 & 2 & 0.067 & 0.067 & 0.117 \\
\hline CoUg16919 & 8 & 0.737 & 0.791 & 0.815 & 4 & 0.235 & 0.683 & 0.653 & 4 & 0.214 & 0.691 & 0.657 \\
\hline CoUg65596 & 9 & 0.789 & 0.853 & 0.867 & 6 & 0.529 & 0.784 & 0.763 & 6 & 0.643 & 0.794 & 0.764 \\
\hline CoUg5643 & 6 & 1.000 & 0.750 & 0.785 & 4 & 0.000 & 0.734 & 0.713 & 4 & 0.000 & 0.751 & 0.724 \\
\hline CoUg3898 & 3 & 0.177 & 0.415 & 0.445 & - & - & - & - & - & - & - & - \\
\hline CoUg12112 & 7 & 0.800 & 0.744 & 0.778 & 5 & 0.118 & 0.562 & 0.565 & 4 & 0.000 & 0.561 & 0.541 \\
\hline CoUg12183 & 3 & 0.111 & 0.298 & 0.34 & - & - & - & - & - & - & - & - \\
\hline CoUg59126 & 6 & 0.947 & 0.626 & 0.683 & 4 & 0.278 & 0.614 & 0.631 & 4 & 0.333 & 0.641 & 0.650 \\
\hline CoUg59697 & 10 & 1.000 & 0.811 & 0.832 & 4 & 0.389 & 0.467 & 0.509 & 3 & 0.286 & 0.442 & 0.475 \\
\hline CoUg59762 & 5 & 0.923 & 0.607 & 0.667 & 5 & 0.722 & 0.759 & 0.747 & 5 & 0.714 & 0.757 & 0.747 \\
\hline CoUg60001 & 4 & 0.900 & 0.472 & 0.566 & 4 & 0.056 & 0.427 & 0.432 & 3 & 0.067 & 0.301 & 0.320 \\
\hline CoUg62529 & 7 & 1.000 & 0.745 & 0.779 & 4 & 0.294 & 0.629 & 0.628 & 3 & 0.143 & 0.601 & 0.586 \\
\hline CoUg68309 & 3 & 0.353 & 0.549 & 0.628 & 4 & 0.765 & 0.758 & 0.711 & 4 & 0.714 & 0.773 & 0.726 \\
\hline CoUg68886 & 4 & 0.579 & 0.607 & 0.654 & 3 & 0.111 & 0.560 & 0.580 & 3 & 0.067 & 0.549 & 0.555 \\
\hline CoUg3005 & 12 & 0.722 & 0.882 & 0.891 & 10 & 0.556 & 0.865 & 0.862 & - & - & - & - \\
\hline CoUg3184 & 5 & 0.474 & 0.739 & 0.775 & 5 & 0.222 & 0.751 & 0.731 & 5 & 0.286 & 0.759 & 0.728 \\
\hline CoUg3222 & 11 & 0.944 & 0.878 & 0.888 & 7 & 0.375 & 0.802 & 0.818 & 7 & 0.385 & 0.791 & 0.809 \\
\hline CoUg3397 & 11 & 0.750 & 0.856 & 0.870 & 8 & 1.000 & 0.873 & 0.849 & 7 & 0.867 & 0.851 & 0.821 \\
\hline CoUg6065 & 3 & 0.158 & 0.580 & 0.653 & 4 & 0.000 & 0.535 & 0.519 & 3 & 0.000 & 0.519 & 0.500 \\
\hline CoUg6948 & 7 & 0.368 & 0.691 & 0.728 & 7 & 0.529 & 0.795 & 0.757 & 6 & 0.500 & 0.712 & 0.689 \\
\hline CoUg7148 & 6 & 0.526 & 0.680 & 0.718 & 5 & 0.353 & 0.779 & 0.767 & 5 & 0.357 & 0.775 & 0.759 \\
\hline Mean & 7.096 & 0.697 & 0.707 & 0.742 & 5.319 & 0.362 & 0.672 & 0.670 & 4.610 & 0.302 & 0.652 & 0.649 \\
\hline
\end{tabular}

$\mathrm{N}=$ number of individuals tested; $N_{\mathrm{A}}=$ number of alleles; $H_{\mathrm{O}}=$ observed heterozygosity; $H_{\mathrm{E}}=$ expected heterozygosity. $\mathrm{PIC}=$ polymorphism information content. 


\section{Putative function of the SSR-containing unigenes}

To determine the function of polymorphic SSR-associated unigenes, the newly developed SSRs were evaluated for associations with genes of known function. The 52 sequences were BLASTed against the GenBank nonredundant database using BLASTx with $E$ value $<1 \times 10^{-5}$. Of the 52 unigenes, 35 showed significant similarities to known genes, including those for an alpha/beta hydrolase fold family protein (CoUg65), an F-box family protein (CoUg3680), a pectate lyase family protein (CoUg7967), a zinc finger family protein (CoUg4364), UDP-galactose/UDP-glucose transporter-related protein (CoUg60001), and others listed in Table 1.

\section{DISCUSSION}

In this study we discovered 6949 SSR-motifs with 2-6 nucleotide repeats from 6042 SSR-containing unique putative transcripts among the 69,798 unigenes in C. oleifera. We found that the most abundant SSR motifs in this tree species identified in this study were AG/ $\mathrm{CT}$ and $\mathrm{AAG} / \mathrm{CTT}$. A similar bias towards $\mathrm{AG}$ and AAG, and against CG repeats, has been reported in EST-SSRs of other plant species (Blanca et al., 2011; Xu et al., 2012; Zhang et al., 2012). According to Gonzalez-Ibeas et al. (2007), this may have resulted from the tendency of $\mathrm{CpG}$ sequences to be methylated, which may potentially inhibit transcription.

We developed 52 polymorphic SSR markers in which all SSR motifs contained 20 or more nucleotides. Of these markers, 47 and 41 can be transferable to two allied species of C. oleifera, C. chekangoleosa and C. japonica, respectively. In addition, the 52 SSR markers potentially encoded functional genes since they were developed from unigenes. Genbank database search identified 35 of the 52 loci putatively coding for functional proteins, therefore these genes may be correlated with seed development in C. oleifera. In comparison with genomic SSR markers, these unigene-derived SSR markers have special features because they are associated with functional genes and may increase the efficiency of marker-assisted selection (Gupta and Rustgi, 2004). These 52 informative unigene-derived SSR markers will be valuable for analyses of genetic variation and marker-assisted selection in breeding programs for C. oleifera, C. chekangoleosa, and C. japonica.

\section{ACKNOWLEDGMENTS}

Research supported by the National Natural Science Foundation of China (Grant \#31100497) and the Key Project of the Chinese Ministry of Education (Grant \#212126). We thank Dr. Xiaolin Lei of the Jiangxi Academy of Forestry for plant sample collection.

\section{REFERENCES}

Blanca J, Cañizares J, Roig C, Ziarsolo P, et al. (2011). Transcriptome characterization and high throughput SSRs and SNPs discovery in Cucurbita pepo (Cucurbitaceae). BMC Genomics 12: 104.

Dutta S, Kumawat G, Singh BP, Gupta DK, et al. (2011). Development of genic-SSR markers by deep transcriptome sequencing in pigeon pea [Cajanus cajan (L.) Millspaugh]. BMC Plant Biol. 11: 17.

Gonzalez-Ibeas D, Blanca J, Roig C, González-To M, et al. (2007). MELOGEN: an EST database for melon functional genomics. BMC Genomics 8: 306.

Gupta PK and Rustgi S (2004). Molecular markers from the transcribed/expressed region of the genome in higher plants. Funct. Integr. Genomics 4: 139-162. 
Gupta SK and Gopalakrishna T (2010). Development of unigene-derived SSR markers in cowpea (Vigna unguiculata) and their transferability to other Vigna species. Genome 53: 508-523.

Huang Y (2013). Population genetic structure and interspecific introgressive hybridization between Camellia meiocarpa and C. oleifera. J. Appl. Ecol. 24: 2345-2352.

Ma JL, Ye H, Rui YK, Chen GC, et al. (2011). Fatty acid composition of Camellia oleifera oil. J. Consum. Prot. Food Safety 6: 9-12.

Marioni JC, Mason CE, Mane SM, Stephens M, et al. (2008). RNA-seq: an assessment of technical reproducibility and comparison with gene expression arrays. Genome Res. 18: 1509-1517.

Mortazavi A, Williams BA, Mccue K, Schaeffer L, et al. (2008). Mapping and quantifying mammalian transcriptomes by RNA-Seq. Nat. Methods 5: 621-628.

Nagalakshmi U, Wang Z, Waern K, Shou C, et al. (2008). The transcriptional landscape of the yeast genome defined by RNA sequencing. Science 320: 1344-1349.

Schuelke M (2000). An economic method for the fluorescent labeling of PCR fragments. Nat. Biotechnol 18: 233-234.

Shao FG (2011). Research on Transcriptome and Gene Expression Profiles involved in Lipid Biosynthesis and Regulation in the Developing Seeds of Camellia oleifera. Doctoral thesis, Central South University of Forestry and Technology, Changsha.

Shi J, Dai XG, Chen YG, Chen JH, et al. (2013). Discovery and experimental analysis of microsatellites in an oil woody plant Camellia chekiangoleosa. Plant Syst. Evol. 299: 1387-1393.

Singh RK, Jena SN, Khan S, Yadav S, et al. (2013). Development, cross-species/genera transferability of novel EST-SSR markers and their utility in revealing population structure and genetic diversity in sugarcane. Gene 524: 309-329.

Wen Q, Xu LC, Gu YC, Huang MR, et al. (2012). Development of polymorphic microsatellite markers in Camellia chekiangoleosa (Theaceae) using 454-ESTs. Am. J. Bot. 99: e203-205.

Xu W, Yang Q, Huai HY and Liu AZ (2012). Development of EST-SSR markers and investigation of genetic relatedness in tung tree. Tree Genet. Genomes 8: 933-940.

Zhang JN, Liang S, Duan JL, Wang J, et al. (2012). De novo assembly and characterisation of the transcriptome during seed development, and generation of genic-SSR markers in peanut (Arachis hypogaea L.). BMC Genomics 13: 90. 